Research Article

\title{
Fault-Tolerant Partition Resolvability of Cyclic Networks
}

\author{
Kamran Azhar $\mathbb{D},{ }^{1}$ Sohail Zafar $\mathbb{D},{ }^{1}$ Agha Kashif $\mathbb{D},{ }^{1}$ and Michael Onyango Ojiema $\mathbb{D}^{2}$ \\ ${ }^{1}$ University of Management and Technology (UMT), Lahore, Pakistan \\ ${ }^{2}$ Masinde Muliro University of Science and Technology, Kakamega, Kenya
}

Correspondence should be addressed to Michael Onyango Ojiema; mojiema@mmust.ac.ke

Received 29 September 2021; Accepted 26 November 2021; Published 16 December 2021

Academic Editor: Gohar Ali

Copyright ( $\odot 2021$ Kamran Azhar et al. This is an open access article distributed under the Creative Commons Attribution License, which permits unrestricted use, distribution, and reproduction in any medium, provided the original work is properly cited.

Graph invariants provide an amazing tool to analyze the abstract structures of networks. The interaction and interconnection between devices, sensors, and service providers have opened the door for an eruption of mobile over the web applications. Structure of web sites containing number of pages can be represented using graph, where web pages are considered to be the vertices, and an edge is a link between two pages. Figuring resolving partition of the graph is an intriguing inquest in graph theory as it has many applications such as sensor design, compound classification in chemistry, robotic navigation, and Internet network. The partition dimension is a graph parameter akin to the concept of metric dimension, and fault-tolerant partition dimension is an advancement in the line of research of partition dimension of the graph. In this paper, we compute fault-tolerant partition dimension of alternate triangular cycle, mirror graph, and tortoise graphs.

\section{Introduction and Basic Terminologies}

Graph theory is an intense region of arithmetic that has capacious variety of implementations in numerous regions of science, such as chemistry, biology, software engineering, and electrical and hardware engineering. Graphing is a powerful tool for representing and understanding objects and their relationships. Currently, online social networks are considered as an essential element for interpersonal relationships where people and smart objects are connected together in smart environments. Theoretical principles of graph theory are applied to practical fields, by determining graph invariants such as vertices, edges, diameter, and degree and mapping them to real-time problems. These invariants are the supporting tools between science and engineering or computational techniques in the fields of chemical, electrical, computer, and telecommunication engineering. One concept that pervades all the graph theory is that of distance and is used in isomorphism testing, graph operations, maximal and minimal problems on connectivity, and diameter. Metric dimension is one of the distance related parameter in graphs that has attracted the attention of several researchers. The generalized version of metric dimension of the graph is unique and an important parameter of graph theory called partition dimension of the graph. It is considered as an applied topic of graph theory and has applications in structure-activity issues in drug design, network discovery and verification [5], pattern recognition and image processing [16], and modelling of chemical substances [14].

In 2000, the concept of partition dimension of graph was initiated by Chartrand et al. as an extension of metric dimension of the graph [6]. Let $\Omega$ be a connected graph of order $n$ with vertex set $V(\Omega)$ and edge set $E(\Omega)$. If two vertices $w, z \in V(\Omega)$, then the length of shortest path between $w$ and $z$ in $\Omega$ is distance between these vertices and is denoted by $\mathrm{d}(w, z)$. The distance between a vertex $z$ and $J \subseteq V(\Omega)$ is defined as $\min \{\mathrm{d}(z, y) \mid y \in J\}$ and is denoted by $\mathrm{d}(z, J)$. For a vertex $z \in V(\Omega), N(z)$ will denote the open neighbourhood of $z$ in $\Omega$, i.e., $N(z)=\{q \in V(\Omega): q$ is adjacent to $z\}$, and closed neighbourhood of $z$ will be denoted by $N[z]=N(z) \cup\{z\}[18]$. Let $\mu=\left\{z_{1}, z_{2}, \ldots, z_{p}\right\}$ be an ordered subset of vertex set of $\Omega$. The representation of $z$ with respect to $\mu$ is $p$ tuple $\left(\mathrm{d}\left(z, z_{1}\right), \mathrm{d}\left(z, z_{2}\right), \ldots, \mathrm{d}\left(z, z_{p}\right)\right)$ and is denoted by $r(z \mid \mu)$. The subset $\mu$ is called a resolving set of $\Omega$ if distinct vertices of $\Omega$ have distinct representations with respect to $\mu$. The metric dimension of $\Omega$ is defined as $\min \{|\mu|: \mu$ is resolving set of $\Omega\}$ and is denoted by $\beta(\Omega)$. Ali 
et al. discussed that path-related graphs have constant metric dimension [1]. Zuo et al. computed constant metric dimension of some generalized convex polytopes [27]. Rehman et al. computed the metric dimension of arithmetic graph of a composite number [20]. In 2021, metric dimension of windmill graph was computed by Singh et al. [24].

In 2008, Hernando et al. initiated the concept of faulttolerant metric dimension of graphs [10]. If, for every pair of distinct vertices $q, z \in V(\Omega)$, there exists at least two vertices $\alpha_{1}, \alpha_{2} \in \mu$ such that $\mathrm{d}\left(q, \delta_{i}\right) \neq \mathrm{d}\left(z, \delta_{i}\right)$ for $i \in\{1,2\}$, then the resolving set $\mu$ of $V(\Omega)$ is called fault tolerant. The faulttolerant metric dimension of $\Omega$ is the minimum cardinality of fault-tolerant resolving set $\mu$ and is denoted by $\beta^{\prime}(\Omega)$. Ahmad et al. computed fault-tolerant metric dimension of $P(n, 2) \odot K_{1}$ graph [3]. Hayat et al. discussed fault-tolerant metric dimension of interconnection networks [9].

Let $\xi=\left\{\xi_{1}, \xi_{2}, \ldots, \xi_{p}\right\}$ be a partition with $p$ partition classes of vertex set of connected graph $\Omega$. The representation of vertex $z$ with respect to partition set $\xi$ is $p$ vector $\left(\mathrm{d}\left(z, \xi_{1}\right), \mathrm{d}\left(z, \xi_{2}\right), \ldots, \mathrm{d}\left(z, \xi_{p}\right)\right)$ denoted by $r(z \mid \xi)$. The partition $\xi$ is called resolving partition of $\Omega$ if representation of all the vertices in $\Omega$ is different. We define the partition dimension of graph $\Omega$ as $\min \{|\xi|$ : $\xi$ is resolving partition of $\Omega\}$ and is denoted by $p d(\Omega)$. Chartrand et al. [6] characterised the graphs having $p d(\Omega)$ to be 2 or $n$. For various classes of connected graphs, the partition dimension has been obtained. For instance, Ayesha et al. computed the partition dimension of trihexagonal $\alpha$ boron nanotube [23]. Mehreen et al. computed the partition dimension of the fullerene graph [15]. Hussain et al. provided the bounds on partition dimension of generalized Mobius ladder [11]. Monica et al. studied the partition dimension problem for certain classes of the series-parallel graph [17]. Chu et al. calculated the sharp bounds for partition dimension of convex polytopes [7]. Wei et al. studied the partition dimension problem for cycle-related graphs [25]. Yero et al. studied the partition dimension of strong product graphs and Cartesian product graphs [26].

Gary et al. and Khuller et al. mentioned the computational complexity of metric dimension of general graphs $[8,13]$. Computation of $p d(\Omega)$ is more complex as it is more harder than computing metric dimension of a graph.

The concept of fault-tolerant partition dimension of the graph was initiated by Salman et al. [22]. Let $\xi=\left\{\xi_{1}\right.$, $\left.\xi_{2}, \ldots, \xi_{p}\right\}$ be a partition with $p$ partition classes of the vertex set of connected graph $\Omega$. The partition $\xi$ is called faulttolerant resolving partition of $\Omega$ if for every pair of distinct vertices $y, z \in V(\Omega)$, and $r(y \mid \xi)$ and $r(z \mid \xi)$ differ by at least two places. The fault-tolerant partition dimension of $\Omega$ is defined as $\min \{|\xi|: \xi$ is fault- tolerant resolving partition of $\Omega\}$ and is denoted by $\mathscr{F}(\Omega)$. Imran et al. characterised that $\mathscr{F}(\Omega)$ of all the graphs of order $n$ is $n-1$ [12]. Kamran et al. computed the $\mathscr{F}(\Omega)$ of homogeneous caterpillar, tadpole, and necklace graphs $[2,4]$. Asim et al. computed $\mathscr{F}(\Omega)$ of circulant graphs with connection set $\{1,2\}$ in [19]. In this paper, we extend this study by considering alternate triangular cycle, mirror graph, and tortoise graphs and show that they have constant fault-tolerant partition dimension.
Chartrand et al. revealed the following basic results on $p d(\Omega)$.

Proposition 1 (see [6]). Let $\Omega$ be a graph; then,

(1) $p d(\Omega) \leq \beta(\Omega)+1$

(2) $p d(\Omega)=2$ if $\Omega=P_{n}$, where $P_{n}$ is a path

(3) $\operatorname{pd}(\Omega)=n$ if $\Omega=K_{n}$, where $K_{n}$ is the complete graph

Salman et al. revealed the following basic results on $\mathscr{F}(\Omega)$.

Proposition 2 (see [21]). For $n \geq 2$,

(1) $p d(\Omega) \leq \mathscr{F}(\Omega)$

(2) $\mathscr{F}(\Omega)=n$ if $\Omega \cong K_{n}$ or $\Omega \cong K_{n}-e$

Proposition 3 (see [22]).

(1) For $n \geq 2, \mathscr{F}(\Omega) \leq \beta^{\prime}(\Omega)+1$

(2) For $n \geq 3,3 \leq \mathscr{F}(\Omega) \leq n$

The remaining part of the paper is structured in the following manner. In Section 2, we are concerned with the computation of $\mathscr{F}\left(A\left(C_{2 n}\right)\right), \mathscr{F}\left(M r\left(P_{n}\right)\right)$, and $\mathscr{F}\left(T_{n}\right)$, where $A\left(C_{2 n}\right), M r\left(P_{n}\right)$, and $T_{n}$ are alternate triangular cycle, mirror graph, and tortoise graphs, respectively. Finally, we conclude the paper in Section 3, by giving future research direction.

\section{Fault-Tolerant Partition Dimension of Alternate Triangular Cycle}

In this section, we compute $\mathscr{F}\left(A\left(C_{2 n}\right)\right)$, where $A\left(C_{2 n}\right)$ is alternate triangular cycle. Vertices of $A\left(C_{2 n}\right)$, for $n \geq 3$, are divided into three sets, $D=\left\{d_{\varrho}: 1 \leq \varrho \leq n\right\}, E=\left\{e_{\varrho}: 1 \leq \varrho \leq\right.$ $n\}$, and $F=\left\{f_{\varrho}: 1 \leq \varrho \leq n\right\}$. An alternate triangular cycle $A\left(C_{2 n}\right)$ is obtained by replacing alternate edge of an even cycle $C_{2 n}$ by $C_{3}$. The set $V\left(A\left(C_{2 n}\right)\right)=D \cup E \cup F$ and $E(A$ $\left.\left(C_{2 n}\right)\right)=\left\{d_{\varrho} e_{\varrho}: \quad 1 \leq \varrho \leq n\right\} \cup\left\{e_{\varrho} d_{\varrho+1}: 1 \leq \varrho \leq n-1\right\} \cup\left\{d_{1} e_{n}\right\}$ $\cup\left\{d_{\varrho} f_{\varrho}: 1 \leq \varrho \leq n\right\} \cup\left\{e_{\varrho} f_{\varrho}: 1 \leq \varrho \leq n\right\}$ are vertex set and edge set of alternate triangular cycle, respectively. Order of $A\left(C_{2 n}\right)$ is $3 n$. The alternate triangular cycle $A\left(C_{12}\right)$ is shown in Figure 1.

We compute $p d\left(A\left(C_{2 n}\right)\right)$ and (HTML translation failed) in the following theorems.

Theorem 1. The partition dimension of alternate triangular cycle $A\left(C_{2 n}\right)$, for $n \geq 2$, is 3 .

Proof. Let $\xi=\left\{\xi_{1}, \xi_{2}, \xi_{3}\right\}$ be a partition with 3 partition classes of vertex set of $A\left(C_{2 n}\right)$ for $n \geq 2$. For $n=2$, we consider $\xi_{1}=\left\{d_{1}\right\}, \xi_{2}=\left\{e_{1}\right\}$, and $\xi_{3}=\left\{d_{2}, e_{2}, f_{1}, f_{2}\right\}$. It can easily be observed that $\xi$ is resolving partition. 


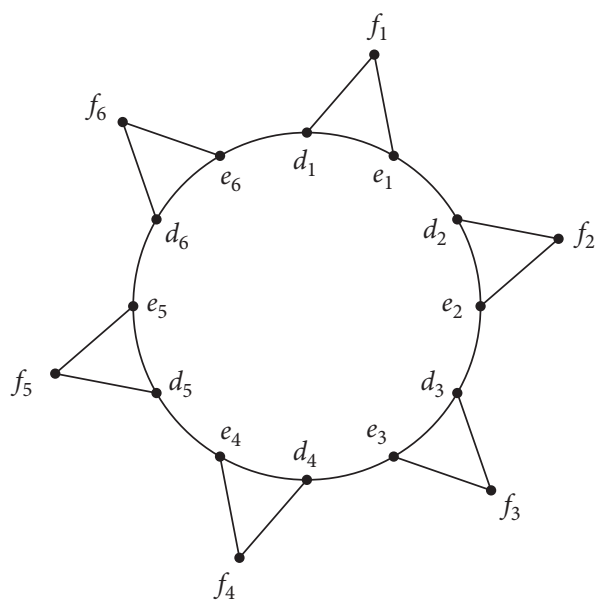

Figure 1: Alternate triangular cycle $A\left(C_{12}\right)$.

Case 1: for $n=3 \delta, \quad \delta \geq 1, \quad r(w \mid \xi)$, where $\xi_{1}=\left\{d_{\varrho}: 1 \leq \varrho \leq n\right\} \cup\left\{e_{\varrho}: 1 \leq \varrho \leq n\right\} \cup\left\{f_{\varrho}: 1 \leq \varrho \leq \delta\right\}$, $\xi_{2}=\left\{f_{\mathrm{\varrho}}: \delta+1 \leq \varrho \leq 2 \delta\right\}$, and $\xi_{3}=\left\{f_{\mathrm{\varrho}}: 2 \delta+1 \leq \mathrm{\varrho} \leq n\right\}$ are shown as follows:

$$
\begin{aligned}
& r\left(d_{\varrho} \mid \xi\right)= \begin{cases}(0,2 \delta-2 \varrho+3,2 \varrho), & 1 \leq \varrho \leq \delta, \\
(0,1, n+\delta-2 \varrho+3), & \delta+1 \leq \varrho \leq 2 \delta, \\
(0,2 \varrho-n-\delta, 1), & 2 \delta+1 \leq \varrho \leq n,\end{cases} \\
& r\left(e_{\varrho} \mid \xi\right)= \begin{cases}(0,2 \delta-2 \varrho+2,2 \varrho+1), & 1 \leq \varrho \leq \delta, \\
(0,1, n+\delta-2 \varrho+2), & \delta+1 \leq \varrho \leq 2 \delta, \\
(0,2 \varrho-n-\delta+1,1), & 2 \delta+1 \leq \varrho \leq n,\end{cases} \\
& r\left(f_{\varrho} \mid \xi\right)= \begin{cases}(0,2 \delta-2 \varrho+3,2 \varrho+1), & 1 \leq \varrho \leq \delta, \\
(1,0, n+\delta-2 \varrho+3), & \delta+1 \leq \varrho \leq 2 \delta, \\
(1,2 \varrho-n-\delta+1,0), & 2 \delta+1 \leq \varrho \leq n .\end{cases}
\end{aligned}
$$

Case 2: for $n=3 \delta+1, \quad \delta \geq 1, \quad r(w \mid \xi)$, where $\xi_{1}=$ $\left\{d_{\varrho}: 1 \leq \varrho \leq n\right\} \cup\left\{e_{\varrho}: 1 \leq \varrho \leq n\right\} \cup\left\{f_{\varrho}: 1 \leq \varrho \leq \delta+1\right\}$, $\xi_{2}=\left\{f_{\varrho}: \delta+2 \leq \varrho \leq 2 \delta+1\right\}$, and $\xi_{3}=\left\{f_{\varrho}: 2 \delta+2 \leq \varrho\right.$ $\leq n\}$ are shown as follows:

$$
\begin{aligned}
& r\left(d_{\varrho} \mid \xi\right)= \begin{cases}(0,2 \delta+2,2), & \varrho=1, \\
(0,2 \delta-2 \varrho+5,2 \varrho), & 2 \leq \varrho \leq \delta+1, \\
(0,1, n+\delta-2 \varrho+4), & \delta+2 \leq \varrho \leq 2 \delta+1, \\
(0,2 \varrho-n-\delta-1,1), & 2 \delta+2 \leq \varrho \leq n,\end{cases} \\
& r\left(e_{\varrho} \mid \xi\right)= \begin{cases}(0,2 \delta-2 \varrho+4,2 \varrho+1), & 1 \leq \varrho \leq \delta, \\
(0,2,2 \delta+2), & \varrho=\delta+1, \\
(0,1, n+\delta-2 \varrho+3), & \delta+2 \leq \varrho \leq 2 \delta+1, \\
(0,2 \varrho-n-\delta, 1), & 2 \delta+2 \leq \varrho \leq n,\end{cases} \\
& r\left(f_{\varrho} \mid \xi\right)= \begin{cases}(0,2 \delta-2 \varrho+5,2 \varrho+1), & 1 \leq \varrho \leq \delta+1, \\
(1,0, n+\delta-2 \varrho+4), & \delta+2 \leq \varrho \leq 2 \delta+1, \\
(1,2 \varrho-n-\delta, 0), & 2 \delta+2 \leq \varrho \leq n .\end{cases}
\end{aligned}
$$

Case 3: for $n=3 \delta+2, \delta \geq 1, r(w \mid \xi)$, where $\xi_{1}=$ $\left\{d_{\varrho}: 1 \leq \varrho \leq n\right\} \cup\left\{e_{\varrho}: 1 \leq \varrho \leq n\right\} \cup\left\{f_{\varrho}: 1 \leq \varrho \leq \delta+1\right\}$, $\xi_{2}=\left\{f_{\varrho}: \delta+2 \leq \varrho \leq 2 \delta+2\right\}$, and $\xi_{3}=\left\{f_{\varrho}: 2 \delta+3 \leq \varrho\right.$ $\leq n\}$ are shown as follows:

$$
\begin{aligned}
& r\left(d_{\varrho} \mid \xi\right)= \begin{cases}(0,2 \delta+2,2) & \varrho=1, \\
(0,2 \delta-2 \varrho+5,2 \varrho) & 2 \leq \varrho \leq \delta+1, \\
(0,1, n+\delta-2 \varrho+5) & \delta+2 \leq \varrho \leq 2 \delta+2, \\
(0,2 \varrho-n-\delta-2,1) & 2 \delta+3 \leq \varrho \leq n,\end{cases} \\
& r\left(e_{\varrho} \mid \xi\right)= \begin{cases}(0,2 \delta-2 \varrho+4,2 \varrho+1) & 1 \leq \varrho \leq \delta+1, \\
(0,1, n+\delta-2 \varrho+4) & \delta+2 \leq \varrho \leq 2 \delta+2, \\
(0,2 \varrho-n-\delta-1,1) & 2 \delta+3 \leq \varrho \leq n,\end{cases} \\
& r\left(f_{\varrho} \mid \xi\right)= \begin{cases}(0,2 \delta-2 \varrho+5,2 \varrho+1), & 1 \leq \varrho \leq \delta+1, \\
(1,0, n+\delta-2 \varrho+5), & \delta+2 \leq \varrho \leq 2 \delta+2, \\
(1,2 \varrho-n-\delta-1,0), & 2 \delta+3 \leq \varrho \leq n .\end{cases}
\end{aligned}
$$

It is obvious from the above distinct representations that $\xi$ is resolving partition of $A\left(C_{2 n}\right)$; therefore, $p d\left(A\left(C_{2 n}\right)\right) \leq 3$. It follows from Proposition $1(\mathrm{~b})$ that $p d\left(A\left(C_{2 n}\right)\right) \geq 3$. This completes the proof.

Theorem 2. The fault-tolerant partition dimension of alternate triangular cycle $A\left(C_{2 n}\right)$, for $n \geq 2$, is 4 .

Proof. In order to prove that $\mathscr{F}\left(A\left(C_{2 n}\right)\right)=4$, first, we show that $\mathscr{F}\left(A\left(C_{2 n}\right)\right) \leq 4$. In this regard, consider $\xi=\left\{\xi_{1}, \xi_{2}, \xi_{3}, \xi_{4}\right\}$ be a partition with 4 partition classes of vertex set of $A\left(C_{2 n}\right)$, for $n \geq 2$. For $n=2$, consider $\xi_{1}=\left\{d_{1}\right\}$, $\xi_{2}=\left\{e_{1}, d_{2}\right\}, \xi_{3}=\left\{e_{2}\right\}$, and $\xi_{4}=\left\{f_{1}, f_{2}\right\}$. It is easy to observe that $\xi$ is fault-tolerant resolving partition.

(1) Case 1: for $n=3 \delta, \quad \delta \geq 1, \quad r(w \mid \xi)$, where $\xi_{1}=\left\{d_{\varrho}: 1 \leq \varrho \leq n\right\} \cup\left\{e_{\varrho}: 1 \leq \varrho \leq n\right\}, \quad \xi_{2}=\left\{f_{\varrho}: 1 \leq\right.$ $\varrho \leq \delta\}, \xi_{3}=\left\{f_{\varrho}: \delta+1 \leq \varrho \leq 2 \delta\right\}$, and $\xi_{4}=\left\{f_{\varrho}: 2 \delta+\right.$ $1 \leq \varrho \leq n\}$ are shown as follows: 


$$
\begin{aligned}
& r\left(d_{\varrho} \mid \xi\right)= \begin{cases}(0,1,2 \delta-2 \varrho+3,2 \varrho), & 1 \leq \varrho \leq \delta, \\
(0,2 \varrho-n+\delta, 1, n+\delta-2 \varrho+3), & \delta+1 \leq \varrho \leq 2 \delta, \\
(0,2 n-2 \varrho+3,2 \varrho-n-\delta, 1), & 2 \delta+1 \leq \varrho \leq n,\end{cases} \\
& r\left(e_{\varrho} \mid \xi\right)= \begin{cases}(0,1,2 \delta-2 \varrho+2,2 \varrho+1), & 1 \leq \varrho \leq \delta, \\
(0,2 \varrho-n+\delta+1,1, n+\delta-2 \varrho+2), & \delta+1 \leq \varrho \leq 2 \delta, \\
(0,2 n-2 \varrho+2,2 \varrho-n-\delta+1,1), & 2 \delta+1 \leq \varrho \leq n,\end{cases} \\
& r\left(f_{\varrho} \mid \xi\right)= \begin{cases}(1,0,2 \delta-2 \varrho+3,2 \varrho+1), & 1 \leq \varrho \leq \delta, \\
(1,2 \varrho-n+\delta+1,0, n+\delta-2 \varrho+3), & \delta+1 \leq \varrho \leq 2 \delta, \\
(1,2 n-2 \varrho+3,2 \varrho-n-\delta+1,0), & 2 \delta+1 \leq \varrho \leq n .\end{cases}
\end{aligned}
$$

(2) Case 2: for $n=3 \delta+1, \quad \delta \geq 1, \quad r(w \mid \xi)$, where $\xi_{1}=\left\{d_{\varrho}: 1 \leq \varrho \leq n\right\} \cup\left\{e_{\varrho}: 1 \leq \varrho \leq n-1\right\}, \quad \xi_{2}=\left\{e_{n}\right\} \cup$

$\left\{f_{\varrho}: 1 \leq \varrho \leq \delta\right\}, \quad \xi_{3}=\left\{f_{\varrho}: \delta+1 \leq \varrho \leq 2 \delta\right\}, \quad$ and $\xi_{4}=\left\{f_{\varrho}: 2 \delta+1 \leq \varrho \leq n\right\}$ are shown as follows:

$$
\begin{aligned}
& r\left(d_{\varrho} \mid \xi\right)= \begin{cases}(0,1,2 \delta-2 \varrho+3,2 \varrho), & 1 \leq \varrho \leq \delta, \\
(0,2 \varrho-n+\delta+1,1, n+\delta-2 \varrho+2), & \delta+1 \leq \varrho \leq 2 \delta, \\
(0,2 n-2 \varrho+1,2 \varrho-n-\delta+1,1), & 2 \delta+1 \leq \varrho \leq n,\end{cases} \\
& r\left(e_{\varrho} \mid \xi\right)= \begin{cases}(0,1,2 \delta-2 \varrho+2,2 \varrho+1), & 1 \leq \varrho \leq \delta, \\
(0,2 \varrho-n+\delta+2,1, n+\delta-2 \varrho+1), & \delta+1 \leq \varrho \leq 2 \delta, \\
(0,2 n-2 \varrho, 2 \varrho-n-\delta+1,1), & 2 \delta+1 \leq \varrho \leq n-1, \\
(1,0,2 \varrho+2,1), & \varrho=n,\end{cases} \\
& r\left(f_{\varrho} \mid \xi\right)= \begin{cases}(1,0,2 \delta-2 \varrho+3,2 \varrho+1), & 1 \leq \varrho \leq \delta, \\
(1,2 \varrho-n+\delta+2,0, n+\delta-2 \varrho+2), & \delta+1 \leq \varrho \leq 2 \delta, \\
(1,2 n-2 \varrho+1,2 \varrho-n-\delta+2,0), & 2 \delta+1 \leq \varrho \leq n .\end{cases}
\end{aligned}
$$

(3) Case 3: for $n=3 \delta+2, \quad \delta \geq 1, \quad r(w \mid \xi)$, where $\xi_{1}=\left\{d_{\varrho}: 1 \leq \varrho \leq n\right\} \cup\left\{e_{\varrho}: 1 \leq \varrho \leq n\right\} \cup\left\{f_{1}\right\}, \quad \xi_{2}=$

$\left\{f_{\varrho}: 2 \leq \varrho \leq \delta+1\right\}, \xi_{3}=\left\{f_{\varrho}: \delta+2 \leq \varrho \leq 2 \delta+2\right\}$, and $\xi_{4}=\left\{f_{\varrho}: 2 \delta+3 \leq \varrho \leq n\right\}$ are shown as follows:

$$
\begin{aligned}
& r\left(d_{\varrho} \mid \xi\right)= \begin{cases}(0,3,2 \delta+2,2), & \varrho=1, \\
(0,1,2 \delta-2 \varrho+5,2 \varrho), & 2 \leq \varrho \leq \delta+1, \\
(0,2 \varrho-n+\delta, 1, n+\delta-2 \varrho+5), & \delta+2 \leq \varrho \leq 2 \delta+2, \\
(0,2 n-2 \varrho+5,2 \varrho-n-\delta-2,1), & 2 \delta+3 \leq \varrho \leq n,\end{cases} \\
& r\left(e_{\varrho} \mid \xi\right)= \begin{cases}(0,2,2 \delta+2,3), & \varrho=1, \\
(0,1,2 \delta-2 \varrho+4,2 \varrho+1), & 2 \leq \varrho \leq \delta+1, \\
(0,2 \varrho-n+\delta+1,1, n+\delta-2 \varrho+4), & \delta+2 \leq \varrho \leq 2 \delta+2, \\
(0,2 n-2 \varrho+4,2 \varrho-n-\delta-1,1), & 2 \delta+3 \leq \varrho \leq n,\end{cases} \\
& r\left(f_{\varrho} \mid \xi\right)= \begin{cases}(0,3,2 \delta+3,3), & \varrho=1, \\
(1,0,2 \delta-2 \varrho+5,2 \varrho+1), & 2 \leq \varrho \leq \delta+1, \\
(1,2 \varrho-n+\delta+1,0, n+\delta-2 \varrho+5), & \delta+2 \leq \varrho \leq 2 \delta+2, \\
(1,2 n-2 \varrho+5,2 \varrho-n-\delta-1,0), & 2 \delta+3 \leq \varrho \leq n .\end{cases}
\end{aligned}
$$


It is obvious from the above distinct representations that $\xi$ is fault-tolerant resolving partition of $A\left(C_{2 n}\right)$, so $\mathscr{F}\left(A\left(C_{2 n}\right)\right) \leq 4$.

Now, we prove that $\mathscr{F}\left(A\left(C_{2 n}\right)\right) \geq 4$. For this, we show that $\mathscr{F}\left(A\left(C_{2 n}\right)\right) \neq 3$. For a contradiction, suppose $\xi=\left\{\xi_{1}, \xi_{2}, \xi_{3}\right\}$ be a fault-tolerant partition basis of $A\left(C_{2 n}\right)$. There will be at least one vertex of degree 3 in one of the partition set $\xi_{1}, \xi_{2}$, or $\xi_{3}$. Without loss of generality, we assume that $d_{0}$ is a vertex of degree 3 that belongs to $\xi_{1}$, and $N\left(d_{\mathrm{\rho}}\right)=\left\{e_{\mathrm{\rho}}, e_{\mathrm{Q}+j}, f_{\mathrm{\rho}}\right\}$. Suppose that $\left|\xi_{1}\right|=1$, and $N\left(d_{\rho}\right) \subseteq \xi_{2} \cup \xi_{3},\left|N\left(d_{\rho}\right) \cap \xi_{2}\right| \geq 2$, or $\left|N\left(d_{0}\right) \cap \xi_{3}\right| \geq 2$. Without loss of generality, we assume that, at least two vertices, $s, t \in N\left(d_{\varrho}\right) \cap \xi_{2}$. As $r(s \mid \xi)=\left(1,0, k_{1}\right)$ and $r(t \mid \xi)=\left(1,0, k_{2}\right)$ are identical at two places, hence, it is a contradiction.

We consider the following cases when $\left|\xi_{1}\right| \geq 2$ and $d_{\varrho} \in \xi_{1}$.

(1) Case 1: if $N\left(d_{0}\right) \cap \xi_{1}=\left\{e_{0}, e_{0+j}, f_{\varrho}\right\}$, then $r\left(d_{\varrho} \mid \xi\right)=\left(0, b_{0}, c_{0}\right), r\left(e_{\varrho} \mid \xi\right)=\left(0, b_{1}, c_{1}\right), r\left(e_{\varrho+j} \mid \xi\right)=$ $\left(0, b_{2}, c_{2}\right)$, and $r\left(f_{\varrho} \mid \xi\right)=\left(0, b_{3}, c_{3}\right)$. As $b_{0}-1 \leq b_{1}$, $b_{2}, b_{3} \leq b_{0}+1$, so using Pigeonhole principle, there will be similarity in the representation of two vertices at two places; hence, it is a contradiction.

(2) Case 2: if two neighbours of $d_{0} \in \xi_{1}$ and one neighbour of $d_{0}$ belongs to $\xi_{2}$, then following cases will arise:

(1) Case 2(a): if $N\left(d_{\varrho}\right) \cap \xi_{1}=\left\{e_{\varrho}, e_{\varrho+j}\right\}$ and one vertex $f_{\varrho} \in \xi_{2}$, then $r\left(d_{\varrho} \mid \xi\right)=\left(0,1, c_{0}\right)$ and $r\left(e_{\varrho} \mid \xi\right)=$ $\left(0,1, c_{1}\right)$. As representation of two vertices has two identical coordinates, hence, it is a contradiction.

(2) Case 2(b): if $N\left(d_{\varrho}\right) \cap \xi_{1}=\left\{e_{\varrho}, f_{\varrho}\right\}$ and one vertex $e_{\varrho+j} \in \xi_{2}$, then, $\quad r\left(d_{\varrho} \mid \xi\right)=\left(0,1, c_{0}\right)$, $r\left(e_{\varrho} \mid \xi\right)=\left(0, b_{1}, c_{1}\right)$, and $r\left(f_{\varrho} \mid \xi\right)=\left(0,2, c_{2}\right)$. Since $1 \leq b_{1} \leq 2$, so representation of two vertices will have two identical coordinates, hence, it is a contradiction.

(3) Case 2(c): if $N\left(d_{\varrho}\right) \cap \xi_{1}=\left\{e_{\varrho}, j, f_{\rho}\right\}$ and one vertex $e_{\varrho} \in \xi_{2}$, then $r\left(d_{\varrho} \mid \xi\right)=\left(0,1, c_{3}\right)$, $r\left(e_{\mathrm{Q}+j} \mid \xi\right)=\left(0, b_{2}, c_{4}\right)$, and $r\left(f_{\varrho} \mid \xi\right)=\left(0,2, c_{5}\right)$. Since $1 \leq b_{2} \leq 2$, so representation of two vertices have two identical coordinates; hence, it is a contradiction.

(3) Case 3: if $N\left(d_{\varrho}\right) \cap \xi_{1}=\left\{e_{\varrho}\right\}$ and two vertices $e_{\varrho+j}, f_{\varrho} \in \xi_{2}, \quad$ then, $\quad r\left(d_{\rho} \mid \xi\right)=\left(0,1, c_{0}\right)$, $r\left(e_{\varrho} \mid \xi\right)=\left(0,1, c_{1}\right), \quad r\left(e_{\varrho+j} \mid \xi\right)=\left(1,0, c_{2}\right), \quad$ and $r\left(f_{\varrho} \mid \xi\right)=\left(1,0, c_{3}\right)$. Since representation of two vertices has two identical coordinates, thus, it is a contradiction.

(4) Case 4: when each of $\xi_{1}, \xi_{2}$, and $\xi_{3}$ contains one neighbour of $d_{0}$, then we have following cases:

(1) Case 4(a): if $N\left(d_{\varrho}\right) \cap \xi_{1}=\left\{e_{\varrho}\right\}, e_{\varrho+j} \in \xi_{2}$, and $f_{\varrho} \in \xi_{3}$, then $\quad r\left(d_{\varrho} \mid \xi\right)=(0,1,1) \quad$ and $r\left(e_{\varrho} \mid \xi\right)=\left(0, c_{1}, 1\right)$, which leads to a contradiction.

(2) Case 4(b): if $N\left(d_{\varrho}\right) \cap \xi_{1}=\left\{e_{0+j}\right\}, e_{\varrho} \in \xi_{2}$ and $f_{\varrho} \in \xi_{3}$, then $r\left(d_{\varrho} \mid \xi\right)=(0,1,1)$ and $r\left(e_{0+j} \mid \xi\right)=(0,2,2)$. Since $N\left(e_{\varrho+j}\right)=\left\{d_{\varrho}, d_{\varrho+j}, f_{\varrho+j}\right\}$, so let $d_{\varrho+j}, f_{\varrho+j} \in \xi_{1}$.
Now, $r\left(f_{\varrho+j} \mid \xi\right)=\left(0, a_{1}, b_{1}\right)$, where $2 \leq a_{1}, b_{1} \leq 3$, and $r\left(d_{\varrho+j} \mid \xi\right)=\left(0, a_{2}, b_{2}\right)$, where $1 \leq a_{2}$ and $b_{2} \leq 3$. It is obvious that representation of two vertices is identical at two places, so a contradiction.

(5) Case 5: if $N\left(d_{0}\right) \cap \xi_{1}=\varnothing$, at least two vertices from $N\left(d_{0}\right)$ belong to $\xi_{2}$. Without loss of generality, we suppose that $e_{\rho}, e_{\rho+j} \in \xi_{2}$; then, $r\left(d_{\rho} \mid \xi\right)=\left(0,1, c_{0}\right)$, $r\left(e_{\rho} \mid \xi\right)=\left(1,0, c_{1}\right)$, and $r\left(e_{\varrho+j} \mid \xi\right)=\left(1,0, c_{2}\right)$. It is again a contradiction.

This discussion concludes the proof; hence, $\mathscr{F}\left(A\left(C_{2 n}\right)\right) \geq 4$.

Example 1. Consider the alternate triangular cycle $A\left(C_{12}\right)$, as shown in Figure 1. If $\xi=\left\{\xi_{1}, \xi_{2}, \xi_{3}, \xi_{4}\right\}$, where $\xi_{1}=\left\{d_{\varrho}: 1 \leq\right.$ $\varrho \leq 6\} \cup\left\{e_{\varrho}: 1 \leq \varrho \leq 6\right\}, \xi_{2}=\left\{f_{1}, f_{2}\right\}, \xi_{3}=\left\{f_{3}, f_{4}\right\}$, and $\xi_{4}=$ $\left\{f_{5}, f_{6}\right\}$ is a partition of $V\left(A\left(C_{12}\right)\right)$, then the representations of vertices of $A\left(C_{12}\right)$ are as follows: $r\left(d_{1} \mid \xi\right)=$ $(0,1,5,2), r\left(d_{2} \mid \xi\right)=(0,1,3,4), r\left(d_{3} \mid \xi\right)=(0,2,1,5), r\left(d_{4} \mid \xi\right)$ $=(0,4,1,3), \quad r\left(d_{5} \mid \xi\right)=(0,5,2,1), \quad r\left(d_{6} \mid \xi\right)=(0,3,4,1)$, $r\left(e_{1} \mid \xi\right)=(0,1,4,3), \quad r\left(e_{2} \mid \xi\right)=(0,1,2,5), \quad r\left(e_{3} \mid \xi\right)=$ $(0,3,1,4), r\left(e_{4} \mid \xi\right)=(0,5,1,2), r\left(e_{5} \mid \xi\right)=(0,4,3,1), r\left(e_{6} \mid \xi\right)$ $=(0,2,5,1), \quad r\left(f_{1} \mid \xi\right)=(1,0,5,3), \quad r\left(f_{2} \mid \xi\right)=(1,0,3,5)$, $r\left(f_{3} \mid \xi\right)=(1,3,0,5), \quad r\left(f_{4} \mid \xi\right)=(1,5,0,3)$, $r\left(f_{5} \mid \xi\right)=(1,5,3,0)$, and $r\left(f_{6} \mid \xi\right)=(1,3,5,0)$.

It can be seen from the above representations that $\xi$ is a fault-tolerant resolving partition of $A\left(C_{12}\right)$.

2.1. Fault-Tolerant Partition Dimension of Mirror Graph. Mirror graph $\operatorname{Mr}\left(P_{n}\right)$, is defined as the disjoint union of graph $\Omega$ and its copy $\Omega$ with additional edges joining each vertex of $\Omega$ to its corresponding vertex in $\Omega$. The set $V\left(\operatorname{Mr}\left(P_{n}\right)\right)=\left\{w_{1}, w_{2}, \ldots, w_{2 n}\right\} \quad$ and $E\left(M r\left(P_{n}\right)\right)=$ $\left\{w_{l} w_{l+1}: 1 \leq l \leq n-1\right\} \cup\left\{w_{n+l} w_{n+l+1}: 1 \leq l \leq n-1\right\} \cup\left\{w_{l}\right.$ $\left.w_{n+l}: 1 \leq l \leq n\right\}$ are vertex set and edge set of mirror graph, respectively. Mirror graph $M r\left(P_{4}\right)$ is shown in Figure 2.

Yero et al. computed the partition dimension of the mirror graph in the following lemma.

Lemma 1 (see [26]). Let $G$ be the mirror graph $\operatorname{Mr}\left(P_{n}\right)$; then, $p d\left(\operatorname{Mr}\left(P_{n}\right)\right)=3$.

The following theorem allows us to compute $\mathscr{F}\left(\operatorname{Mr}\left(P_{n}\right)\right)$.

Theorem 3. For every $n \geq 2$,

$$
\mathscr{F}\left(M r\left(P_{n}\right)\right)= \begin{cases}3, & \text { if } n \text { is } 2, \\ 4, & \text { otherwise. }\end{cases}
$$

Proof. Let $\xi=\left\{\xi_{1}, \xi_{2}, \xi_{3}\right\}$ be a partition set of vertices of $\operatorname{Mr}\left(P_{n}\right)$ for $n=2$. Considering $\xi_{1}=\left\{w_{1}, w_{2}\right\}, \xi_{2}=\left\{w_{3}\right\}$, and $\xi_{3}=\left\{w_{4}\right\}$, it is easy to observe that $\xi$ is fault-tolerant resolving partition of $\operatorname{Mr}\left(P_{n}\right)$.

Now, for $n \geq 3$, consider $\xi=\left\{\xi_{1}, \xi_{2}, \xi_{3}, \xi_{4}\right\}$ be a partition with 4 partition classes of vertices of $\operatorname{Mr}\left(P_{n}\right) . r(w \mid \xi)$, 


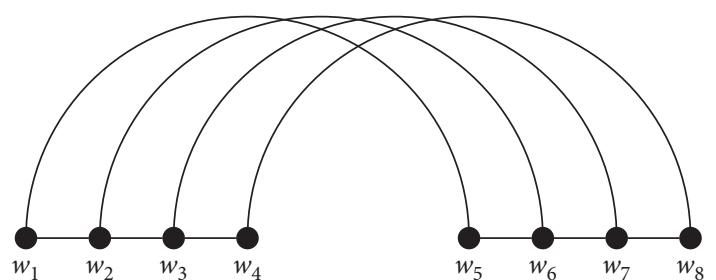

Figure 2: Mirror graph $M r\left(P_{4}\right)$.

considering $\xi_{1}=\left\{w_{j}: 1 \leq j \leq n-1\right\}, \xi_{2}=\left\{w_{n}\right\}, \xi_{3}=\left\{w_{j}: n+\right.$ $1 \leq j \leq 2 n-1\}$, and $\xi_{4}=\left\{w_{2 n}\right\}$, are as follows:

$$
r\left(w_{\varrho} \mid \xi\right)= \begin{cases}(0, n-\varrho, 1, n-\varrho+1) & \text { for } 1 \leq \varrho \leq n-1, \\ (1,0,2,1) & \text { for } \varrho=n, \\ (1,0,2 n-\varrho+1,2 n-\varrho) & \text { for } n+1 \leq \varrho \leq 2 n-1, \\ (2,1,1,0) & \text { for } \varrho=2 n .\end{cases}
$$

As all the above representations are different, so $\xi$ is fault-tolerant resolving partition of $\operatorname{Mr}\left(P_{n}\right)$; therefore, $\mathscr{F}\left(\operatorname{Mr}\left(P_{n}\right)\right) \leq 4$.

Now, we prove that $\mathscr{F}\left(M r\left(P_{n}\right)\right) \geq 4$; for this, we show that $\mathscr{F}\left(\operatorname{Mr}\left(P_{n}\right)\right) \neq 3$. For contradiction, let $\xi=\left\{\xi_{1}, \xi_{2}, \xi_{3}\right\}$ be a fault-tolerant partition basis of $M r\left(P_{n}\right)$. One of the partition sets contains at least one vertex of degree 3 . Without loss of generality, we assume that $w_{\tau}$ is a vertex of degree 3 that belongs to $\xi_{1}$, and $N\left(w_{\tau}\right)=\left\{w_{p}, w_{q}, w_{r}\right\}$. Suppose that $\left|\xi_{1}\right|=1 \quad$ and $\quad N\left(w_{\tau}\right) \subseteq \xi_{2} \cup \xi_{3}, \quad\left|N\left(w_{\tau}\right) \cap \xi_{2}\right| \geq 2$, or $\left|N\left(w_{\tau}\right) \cap \xi_{3}\right| \geq 2$. Without loss of generality, we assume that at least two vertices $e_{1}, e_{2} \in N\left(w_{\tau}\right) \cap \xi_{2}$. As $r\left(e_{1} \mid \xi\right)=\left(1,0, k_{1}\right)$ and $r\left(e_{2} \mid \xi\right)=\left(1,0, k_{2}\right)$ have two identical coordinates, so it is a contradiction. Now, we discuss the following cases when $\left|\xi_{1}\right| \geq 2$ and $w_{\tau} \in \xi_{1}$.

(1) Case 1: if $N\left(w_{\tau}\right) \cap \xi_{1}=\left\{w_{p}, w_{q}, w_{r}\right\}$, then $r\left(w_{\tau} \mid \xi\right)=\left(0, b_{0}, c_{0}\right), \quad r\left(w_{p} \mid \xi\right)=\left(0, b_{1}, c_{1}\right)$, $r\left(w_{q} \mid \xi\right)=\left(0, b_{2}, c_{2}\right)$, and $r\left(w_{r} \mid \xi\right)=\left(0, b_{3}, c_{3}\right)$. As $b_{0}-1 \leq b_{1}, b_{2}, b_{3} \leq b_{0}+1$, so using Pigeonhole principle, representation of two vertices have two identical coordinates; therefore, it is a contradiction.

(2) Case 2: if $N\left(w_{\tau}\right) \cap \xi_{1}=\left\{w_{p}, w_{q}\right\}$ and one vertex $w_{r} \in \xi_{2}$, then $\quad r\left(w_{\tau} \mid \xi\right)=\left(0,1, c_{0}\right)$, $r\left(w_{p} \mid \xi\right)=\left(0, b_{1}, c_{1}\right), \quad r\left(w_{q} \mid \xi\right)=\left(0, b_{2}, c_{2}\right), \quad$ and $r\left(w_{r} \mid \xi\right)=\left(1,0, c_{3}\right)$. Since $1 \leq b_{1}, b_{2} \leq 2$, so there will be similarity in the representation of two vertices at two places from $\xi_{1}$ and $\xi_{2}$; hence, it is a contradiction.

(3) Case 3: if $N\left(w_{\tau}\right) \cap \xi_{1}=\left\{w_{p}\right\}$ and two vertices $w_{q}, w_{r} \in \xi_{2}$, then $r\left(w_{\tau} \mid \xi\right)=\left(0,1, c_{0}\right)$, $r\left(w_{p} \mid \xi\right)=\left(0, b_{1}, c_{1}\right), \quad r\left(w_{q} \mid \xi\right)=\left(1,0, c_{2}\right), \quad$ and $r\left(w_{r} \mid \xi\right)=\left(1,0, c_{3}\right)$. Since $r\left(w_{q} \mid \xi\right)$ and $r\left(w_{r} \mid \xi\right)$ have two identical coordinates, therefore, it is a contradiction.

(4) Case 4: if $N\left(w_{\tau}\right) \cap \xi_{1}=\left\{w_{p}\right\}, w_{q} \in \xi_{2}$, and $w_{r} \in \xi_{3}$, then $N\left(w_{p}\right)=\left\{w_{\tau}, s_{1}\right\}, N\left(w_{q}\right)=\left\{w_{\tau}, s_{1}, s_{2}\right\}$, and
$N\left(w_{r}\right)=\left\{w_{\tau}, s_{2}, s_{3}\right\}$. Let $s_{1} \in \xi_{1}, s_{2} \in \xi_{2}$ and $s_{3} \in \xi_{3}$, then $r\left(w_{\tau} \mid \xi\right)=(0,1,1)$ and $r\left(s_{1} \mid \xi\right)=\left(0,1, c_{1}\right)$, which leads to a contradiction. Now, let $s_{1} \in \xi_{1}$, $s_{3} \in \xi_{2}$, and $s_{2} \in \xi_{3}$; then, $r\left(w_{\tau} \mid \xi\right)=(0,1,1)$ and $r\left(s_{1} \mid \xi\right)=\left(0,1, c_{1}\right)$, which leads to a contradiction.

(5) Case 5: if $N\left(w_{\tau}\right) \cap \xi_{1}=\varnothing$ and at least two vertices from $N\left(w_{\tau}\right)$ belong to $\xi_{2}$, without loss of generality, we suppose that $w_{p}, w_{q} \in \xi_{2}$; then, $r\left(w_{\tau} \mid \xi\right)=\left(0,1, c_{0}\right), \quad r\left(w_{p} \mid \xi\right)=\left(1,0, c_{1}\right), \quad$ and $r\left(w_{q} \mid \xi\right)=\left(1,0, c_{2}\right)$. Again $r\left(w_{p} \mid \xi\right)$ and $r\left(w_{q} \mid \xi\right)$ have two identical coordinates, a contradiction.

The above discussions show that $\mathscr{F}\left(M r\left(P_{n}\right)\right) \geq 4$, which completes the proof.

Example 2. Consider the mirror graph $M r\left(P_{4}\right)$, as shown in Figure 2. If $\xi=\left\{\xi_{1}, \xi_{2}, \xi_{3}, \xi_{4}\right\}$, where $\xi_{1}=\left\{w_{1}, w_{2}, w_{3}\right\}$, $\xi_{2}=\left\{w_{4}\right\}, \xi_{3}=\left\{w_{5}, w_{6}, w_{7}\right\}$, and $\xi_{4}=\left\{w_{8}\right\}$ is a partition of $V\left(M r\left(P_{4}\right)\right)$, then the representations of vertices of $\operatorname{Mr}\left(P_{4}\right)$ are as follows: $r\left(w_{1} \mid \xi\right)=(0,3,1,4), r\left(w_{2} \mid \xi\right)=(0,2,1,3)$, $r\left(w_{3} \mid \xi\right)=(0,1,1,2), \quad r\left(w_{4} \mid \xi\right)=(1,0,2,1), \quad r\left(w_{5} \mid \xi\right)=$ $(1,4,0,3), r\left(w_{6} \mid \xi\right)=(1,3,0,2), r\left(w_{7} \mid \xi\right)=(1,2,0,1)$, and $r\left(w_{8} \mid \xi\right)=(2,1,1,0)$. It is obvious from the above representations that $\xi$ is a fault-tolerant resolving partition of $\operatorname{Mr}\left(P_{4}\right)$.

2.2. Fault-Tolerant Partition Dimension of Tortoise Graph. The tortoise graph, denoted by $T_{n}$, has vertex set $V\left(T_{n}\right)=$ $\left\{w_{1}, w_{2}, \ldots, w_{n}\right\}$ and edge set $E\left(T_{n}\right)=\left\{w_{j} w_{j+1}\right.$ : $1 \leq j \leq n-1\} \cup\left\{w_{j} w_{n-j+1}: 1 \leq j \leq\lfloor n / 2\rfloor\right\}$. Tortoise graph $T_{7}$ is shown in Figure 3.

The following theorems allow us to compute $p d\left(T_{n}\right)$ and $\mathscr{F}\left(T_{n}\right)$.

Theorem 4. For every $n \geq 3$, where $n=2 \delta+1$ and $\delta \geq 1$, $p d\left(T_{n}\right)=3$.

Proof. Let $\xi=\left\{\xi_{1}, \xi_{2}, \xi_{3}\right\}$ be a partition set of $V\left(T_{n}\right)$ for $n \geq 3$. $r(w \mid \xi)$ with respect to $\xi_{1}=\left\{w_{i}: 1 \leq i \leq n-1 / 2\right\}$, $\xi_{2}=\left\{w_{n+1 / 2}\right\}$, and $\xi_{3}=\left\{w_{i}: n+3 / 2 \leq i \leq n\right\}$ are as follows:

$$
r\left(w_{\varrho} \mid \xi\right)= \begin{cases}(0, \delta-\varrho+1,1), & \text { for } 1 \leq \varrho \leq \delta, \\ (1,0,1), & \text { for } \varrho=\delta+1, \\ (1,0, \varrho-\delta-1), & \text { for } \delta+2 \leq \varrho \leq n\end{cases}
$$

It is obvious from the above distinct representations that $\xi$ is resolving partition of $T_{n}$; therefore, $p d\left(T_{n}\right) \leq 3$. It follows by Proposition 1(b) that $p d\left(T_{n}\right) \geq 3$. Hence, $p d\left(T_{n}\right)=3$.

Theorem 5. For every $n \geq 3$, where $n=2 \delta+1$ and $\delta \geq 1$,

$$
\mathscr{F}\left(T_{n}\right)= \begin{cases}3, & \text { if } n \text { is } 3, \\ 4, & \text { otherwise. }\end{cases}
$$




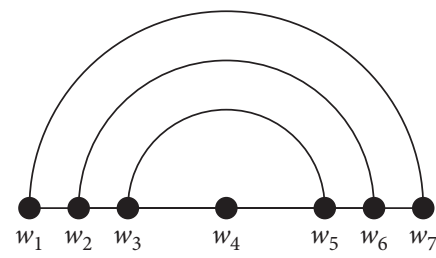

Figure 3: Tortoise graph $T_{7}$.

Proof. Let $\xi=\left\{\xi_{1}, \xi_{2}, \xi_{3}\right\}$ be a partition set of $V\left(T_{n}\right)$ for $n=3$. Considering $\xi_{1}=\left\{w_{1}\right\}, \xi_{2}=\left\{w_{2}\right\}$, and $\xi_{3}=\left\{w_{3}\right\}$, it is easy to see that $\xi$ is fault-tolerant resolving partition of $T_{n}$. Now, consider $\xi=\left\{\xi_{1}, \xi_{2}, \xi_{3}, \xi_{4}\right\}$ be a partition with 4 partition classes of vertex set of $T_{n}$ for $n=2 \delta+1$, where $\delta \geq 2$. $r(w \mid \xi)$ considering $\xi_{1}=\left\{w_{i}: 1 \leq i \leq n-1 / 2\right\}, \quad \xi_{2}=\left\{w_{i}: n+\right.$ $1 / 2 \leq i \leq n-2\}, \xi_{3}=\left\{w_{n-1}\right\}$ and $\xi_{4}=\left\{w_{n}\right\}$ are as follows:

$$
r\left(w_{\varrho} \mid \xi\right)= \begin{cases}(0,2,2,1), & \text { for } \varrho=1, n=5, \\ (0,1,1,2), & \text { for } \varrho=2, n=5, \\ (0,3,2,1), & \text { for } \varrho=1, n \geq 7, \\ (0,2,1,2), & \text { for } \varrho=2, n \geq 7, \\ (0,1, \varrho-1, \varrho), & \text { for } 3 \leq \varrho \leq \delta, \\ (1,0, n-\varrho-1, n-\varrho), & \text { for } \delta+1 \leq \varrho \leq n-2, \\ (1,1,0,1), & \text { for } \varrho=n-1, \\ (1,2,1,0), & \text { for } \varrho=n .\end{cases}
$$

Distinct representations given above show that $\xi$ is faulttolerant resolving partition of $T_{n}$; therefore, $\mathscr{F}\left(T_{n}\right) \leq 4$.

Now, we prove that $\mathscr{F}\left(T_{n}\right) \geq 4$. For this, we show that $\mathscr{F}\left(T_{n}\right) \neq 3$. For contradiction, suppose $\xi=\left\{\xi_{1}, \xi_{2}, \xi_{3}\right\}$ be a fault-tolerant partition basis of $T_{n}$. One of the partition sets contains at least one vertex of degree 3. Without loss of generality, we assume that $w_{\tau}$ is a vertex of degree 3 that belongs to $\xi_{1}$, and $N\left(w_{\tau}\right)=\left\{w_{p}, w_{q}, w_{r}\right\}$. Suppose that $\left|\xi_{1}\right|=1, \quad$ and $\quad N\left(w_{\tau}\right) \subseteq \xi_{2} \cup \xi_{3}, \quad\left|N\left(w_{\tau}\right) \cap \xi_{2}\right| \geq 2$, or $\left|N\left(w_{\tau}\right) \cap \xi_{3}\right| \geq 2$. Without loss of generality, we assume at least two vertices $f_{1}, f_{2} \in N\left(w_{\tau}\right) \cap \xi_{2}$. As $r\left(\left|f_{1}\right| \xi\right)=\left(1,0, k_{1}\right)$ and $r\left(f_{2} \mid \xi\right)=\left(1,0, k_{2}\right)$ has two identical coordinates, therefore, it is a contradiction. Now, we consider the following cases when $\left|\xi_{1}\right| \geq 2$ and $w_{\tau} \in \xi_{1}$.

(1) Case 1: if $N\left(w_{\tau}\right) \cap \xi_{1}=\left\{w_{p}, w_{q}, w_{r}\right\}$, then $r\left(w_{\tau} \mid \xi\right)=\left(0, b_{0}, c_{0}\right), r\left(\left|w_{p}\right| \xi\right)=\left(0, b_{1}, c_{1}\right), r\left(w_{q} \mid \xi\right)$ $=\left(0, b_{2}, c_{2}\right)$, and $r\left(w_{r} \mid \xi\right)=\left(0, b_{3}, c_{3}\right)$. As $b_{0}-1 \leq$ $b_{1}, b_{2}, b_{3} \leq b_{0}+1$, so using Pigeonhole principle, there will be similarity in the representation of two vertices at two places, hence a contradiction.

(2) Case 2: if $N\left(w_{\tau}\right) \cap \xi_{1}=\left\{w_{p}, w_{q}\right\}$ and one vertex $w_{r} \in \xi_{2}$, then $\quad r\left(w_{\tau} \mid \xi\right)=\left(0,1, c_{0}\right), \quad r\left(w_{p} \mid \xi\right)=$ $\left(0, b_{1}, c_{1}\right), \quad r\left(w_{q} \mid \xi\right)=\left(0, b_{2}, c_{2}\right), \quad$ and $\quad r\left(w_{r} \mid \xi\right)=$ $\left(1,0, c_{3}\right)$. Since $1 \leq b_{1}, b_{2} \leq 2$, so representation of two vertices will have two identical coordinates, hence a contradiction.

(3) Case 3: if $N\left(w_{\tau}\right) \cap \xi_{1}=\left\{w_{p}\right\}$ and two vertices $w_{q}, w_{r} \in \xi_{2}$, then $r\left(w_{\tau} \mid \xi\right)=\left(0,1, c_{0}\right), \quad r\left(w_{p} \mid \xi\right)=$ $\left(0, b_{1}, c_{1}\right), \quad r\left(w_{q} \mid \xi\right)=\left(1,0, c_{2}\right), \quad$ and $\quad r\left(w_{r} \mid \xi\right)$ $=\left(1,0, c_{3}\right)$. Since, representation of two vertices has two identical coordinates, thus, it is a contradiction.

(4) Case 4: if $N\left(w_{\tau}\right) \cap \xi_{1}=\left\{w_{p}\right\}, w_{q} \in \xi_{2}$ and $w_{r} \in \xi_{3}$.

(1) For $n=5, N\left(w_{p}\right)=\left\{w_{\tau}, s_{1}\right\}, N\left(w_{q}\right)=\left\{w_{\tau}, w_{r}\right\}$, and $N\left(w_{r}\right)=\left\{w_{\tau}, w_{q}, s_{1}\right\}$. Let $\quad s_{1} \in \xi_{1} ; \quad$ then $r\left(w_{\tau} \mid \xi\right)=(0,1,1), \quad r\left(w_{p} \mid \xi\right)=(0,2,2), \quad$ and $r\left(s_{1} \mid \xi\right)=\left(0, q_{1}, 1\right)$, which leads to a contradiction.

(2) For $n \geq 7, N\left(w_{p}\right)=\left\{w_{\tau}, s_{1}, s_{2}\right\}, N\left(w_{q}\right)=\left\{w_{\tau}, s_{3}\right\}$, and $N\left(w_{r}\right)=\left\{w_{\tau}, s_{2}, s_{3}\right\}$. Let, $s_{1}, s_{2} \in \xi_{1}$ and $s_{3} \in \xi_{2}$; then, $\quad r\left(w_{\tau} \mid \xi\right)=(0,1,1), \quad r\left(w_{p} \mid \xi\right)=(0,2,2)$, $r\left(s_{1} \mid \xi\right)=\left(0, q_{1}, g_{1}\right)$, and $r\left(s_{2} \mid \xi\right)=\left(0, q_{2}, 1\right)$, which leads to a contradiction. Now, let $s_{1}, s_{2} \in \xi_{1}$ and $s_{3} \in \xi_{3}$; then, $r\left(w_{\tau} \mid \xi\right)=(0,1,1), r\left(w_{p} \mid \xi\right)=(0,2,2)$, $r\left(s_{1} \mid \xi\right)=\left(0, q_{1}, g_{1}\right)$, and $r\left(s_{2} \mid \xi\right)=\left(0, q_{2}, 1\right)$, which leads to a contradiction.

(5) Case 5: if $N\left(w_{\tau}\right) \cap \xi_{1}=\varnothing, \xi_{2}$ contains at least two neighbours of $w_{\tau}$. Without loss of generality, we suppose that $w_{p}, w_{q} \in \xi_{3}$; then, $r\left(w_{\tau} \mid \xi\right)=\left(0, c_{0}, 1\right)$, $r\left(w_{p} \mid \xi\right)=\left(1, c_{1}, 0\right)$, and $r\left(w_{q} \mid \xi\right)=\left(1, c_{2}, 0\right)$. Again $r\left(w_{p} \mid \xi\right)$ and $r\left(w_{q} \mid \xi\right)$ have two identical coordinates; hence, it is a contradiction.

The above discussion follows that $\mathscr{F}\left(T_{n}\right) \geq 4$. Thus, $\mathscr{F}\left(T_{n}\right)=4$.

Example 3. Consider the tortoise graph $T_{7}$, as shown in Figure 3. If $\xi=\left\{\xi_{1}, \xi_{2}, \xi_{3}, \xi_{4}\right\}$, where $\xi_{1}=\left\{w_{1}, w_{2}, w_{3}\right\}$, $\xi_{2}=\left\{w_{4}, w_{5}\right\}, \xi_{3}=\left\{w_{6}\right\}$, and $\xi_{4}=\left\{w_{7}\right\}$ is a partition of $V\left(T_{7}\right)$, then $r(w \mid \xi)$ are as follows: $r\left(w_{1} \mid \xi\right)=(0,3,2,1)$, $r\left(w_{2} \mid \xi\right)=(0,2,1,2), \quad r\left(w_{3} \mid \xi\right)=(0,1,2,3), \quad r\left(w_{4} \mid \xi\right)=$ $(1,0,2,3), r\left(w_{5} \mid \xi\right)=(1,0,1,2), r\left(w_{6} \mid \xi\right)=(1,1,0,1)$, and $r\left(w_{7} \mid \xi\right)=(1,2,1,0)$. The above distinct representations verify that $\xi$ is a fault-tolerant resolving partition of $T_{7}$.

\section{Conclusion}

In this paper, authors conclude that $p d(\Omega)$ of alternate triangular cycle and tortoise graph for $n \geq 2$ is 3 . $\mathscr{F}(\Omega)$ of alternate triangular cycle, mirror graph, and tortoise graph for $n \geq 2$ is between 3 and 4 . The obtained results led us to the conclusion that the discussed cyclic networks have constant partition and fault-tolerant partition dimension. $p d(\Omega)$ and $\mathscr{F}(\Omega)$ of these graphs are independent of the number of vertices of the graph. Future research can focus on computing the fault-tolerant partition dimension of alternate square and alternate pentagonal cycle.

The significance of the current work can be seen in optimization related to resource management, routing problem, and supply chain problem. These require to group certain nodes in the network to optimally approach the 
whole network. The least number of grouping required in such scenario can be realised as partition dimension problem. Also, the least number of grouping required to approach the whole network in case of inaccessibility of one of the group relates to fault-tolerant partition dimension of the graph.

\section{Data Availability}

All data used to support the findings of the study are included within this article and can be obtained from the corresponding author upon request.

\section{Conflicts of Interest}

The authors declare that they have no conflicts of interest.

\section{References}

[1] M. Ali, M. T. Rahim, and G. Ali, "On path related graphs with constant metric dimension," Utilitas Mathematica, vol. 88, pp. 203-209, 2012.

[2] K. Azhar, S. Zafar, and A. Kashif, "On fault-tolerent partition dimension of homogeneous caterpillar graphs," Mathematical Problems in Engineering, vol. 2021, p. 7, Article ID 7282245, 2021.

[3] Z. Ahmad, M. A. Chaudhary, A. Q. Baig, and M. A. Zahid, "Fault-tolerant metric dimension of $\mathrm{P}(\mathrm{n}, 2) \odot \mathrm{K} 1$ graph," Journal of Discrete Mathematical Sciences and Cryptography, vol. 24, no. 2, pp. 647-656, 2021.

[4] K. Azhar, S. Zafar, A. Kashif, and Z. Zahid, "On fault-tolerant partition dimension of graphs," Journal of Intelligent and Fuzzy Systems, vol. 40, no. 1, pp. 1129-1135, 2021.

[5] Z. Beerliová, F. Eberhard, T. Erlebach et al., "Network discovery and verification," IEEE Journal on Selected Areas in Communications, vol. 24, no. 12, pp. 2168-2181, 2006.

[6] G. Chartrand, E. Salehi, and P. Zhang, "The partition dimension of a graph," Aequationes Mathematicae, vol. 59, no. 1 , pp. $45-54,2000$.

[7] Y.-M. Chu, M. F. Nadeem, M. Azeem, and M. K. Siddiqui, "On sharp bounds on partition dimension of convex polytopes," IEEE Access, vol. 8, pp. 224781-224790, 2020.

[8] M. R. Garey and D. S. Johnson, Computers and Intractability, A Guide to the Theory of NP-Completeness, Freeman, New York, NY, USA, 1979.

[9] S. Hayat, A. Khan, M. Y. H. Malik, M. Imran, and M. K. Siddiqui, "Fault-tolerant metric dimension of interconnection networks," IEEE Access, vol. 8, pp. 145435-145445, 2020.

[10] C. Hernando, M. Mora, P. J. Slater, and D. R. Wood, "Faulttolerant metric dimension of graphs," in Proceedings of the International Conference on Convexity in Discrete Structures, pp. 81-85, Tiruchirappalli, India, January 2008.

[11] Z. Hussain, J. A. Khan, M. Munir, M. S. Saleem, and Z. Iqbal, "Sharp bounds for partition dimension of generalized Möbius ladders," Open Mathematics, vol. 16, no. 1, pp. 1283-1290, 2018.

[12] I. Javaid, M. Salman, and M. A. Chaudhry, "Graphs of order $n$ with fault-tolerant partition dimension $n-1$," UPB Scientific Bull. Series A, vol. 76, no. 3, 2014.

[13] S. Khuller, B. Raghavachari, and A. Rosenfeld, "Landmarks in graphs," Discrete Applied Mathematics, vol. 70, no. 3, pp. 217-229, 1996.
[14] S. Khuller, B. Raghavachari, and A. Rosenfeld, Location in Graphs, Technical Report CS-TR-3326, University of Maryland at Colleg Park, College Park, MD, USA, 1994.

[15] N. Mehreen, R. Farooq, R. Farooq, and S. Akhter, "On partition dimension of fullerene graphs," AIMS Mathematics, vol. 3, no. 3, pp. 343-352, 2018.

[16] R. A. Melter and I. Tomescu, "Metric bases in digital geometry," Computer Vision, Graphics, and Image Processing, vol. 25, no. 1, pp. 113-121, 1984.

[17] C. M. Mohan, S. Santhakumar, M. Arockiaraj, and J.-B. Liu, "Partition dimension of certain classes of series parallel graphs," Theoretical Computer Science, vol. 778, pp. 47-60, 2019.

[18] A. Estrada-Moreno, "On the k-partition dimension of graphs," Theoretical Computer Science, vol. 806, pp. 42-52, 2020.

[19] A. Nadeem, A. Kashif, S. Zafar, and Z. Zahid, "On 2-partition dimension of the circulant graphs," Journal of Intelligent and Fuzzy Systems, vol. 40, no. 5, pp. 9493-9503, 2021.

[20] S. u. Rehman, M. Imran, and I. Javaid, "On the metric dimension of arithmetic graph of a composite number," Symmetry, vol. 12, no. 4, Article ID 607, 2020.

[21] M. Salman, I. Javaid, and M. Chaudhry, "Fault-tolerant metric and partition dimension of graphs," Utilitas Mathematica, vol. 83, pp. 187-199, 2010.

[22] M. Salman, I. Javaid, M. Chaudhry, and S. Shokat, "Faulttolerance in resolvability," Utilitas Mathematica, vol. 80, pp. 263-275, 2009.

[23] A. Shabbir and M. Azeem, "On the partition dimension of trihexagonal $\alpha$-boron nanotube," IEEE Access, vol. 9, pp. 55644-55653, 2021.

[24] P. Singh, S. Sharma, S. Sharma, S. K. Sharma, and V. K. Bhat, "Metric dimension and edge metric dimension of windmill graphs," AIMS Mathematics, vol. 6, no. 9, pp. 9138-9153, 2021.

[25] C. Wei, M. F. Nadeem, H. M. A. Siddiqui, M. Azeem, J. B. Liu, and A. Khalil, "On partition dimension of some cycle-related graphs," Mathematical Problems in Engineering, vol. 2021, p. 8, Article ID 4046909, 2021.

[26] I. González Yero, M. Jakovac, D. Kuziak, and A. Taranenko, "The partition dimension of strong product graphs and Cartesian product graphs," Discrete Mathematics, vol. 331, pp. 43-52, 2014.

[27] X. Zuo, A. Ali, G. Ali, M. K. Siddiqui, M. T. Rahim, and A. Asare-Tuah, "On constant metric dimension of some generalized convex polytopes," Jurnal Matematika, vol. 2021, 2021. 